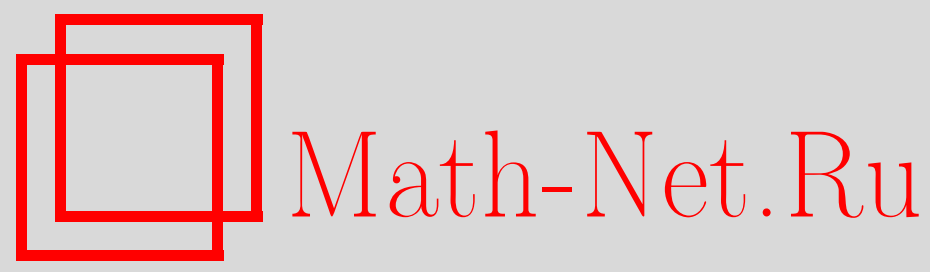

Д. А. Толстоногов, О минимуме в вариационных эллиптических задачах без предположений выпуклости, Матем. заметки, 1999, том 65, выпуск 1, 130-142

DOI: https://doi.org/10.4213/mzm1034

Использование Общероссийского математического портала Math-Net.Ru подразумевает, что вы прочитали и согласны с пользовательским соглашением http://www.mathnet.ru/rus/agreement

Параметры загрузки:

IP : 54.147 .182 .235

26 апреля 2023 г., 15:42:05

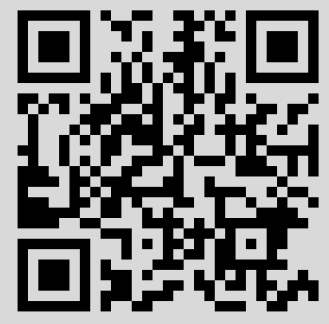




\title{
О МИНИМУМЕ В ВАРИАЦИОННЫХ ЭЛЛИПТИЧЕСКИХ ЗАДАЧАХ БЕЗ ПРЕДПОЛОЖЕНИЙ ВЫПУКЛОСТИ
}

\author{
Д. А. Толстоногов
}

Доказьвается абстрактная теорема существования минимума функционала вида

$$
G(y)+\int_{\Omega} h(x, u(x)) d x
$$

при ограничениях типа неравенств, где отображение $G(y)$ вогнуто, а функция $h(x, u)$ не является выпуклой по $u$, на решениях систем, описьваемых линейными эллиптическими операторами. На примерах дается конкретизация этой теоремы для задач вариационного исчисления и оптимального управления.

Библиография: 18 названий.

Введение. Вопросы сушествования решений в задачах вариационного исчисления и оптимального управления, как правило, после их переформулировки в абстрактной форме сводятся к существованию минимума у полунепрерывного снизу в слабой топологии функционала на слабо компактном множестве соответствующего функционального пространства. Такой подход обычно назьвают прямым. Кроме соответствующих условий роста достаточньм условием полунепрерывности снизу в слабой топологии интегрального функционала в подходящем функциональном пространстве является вьпуклость подынтегральной функции по соответствующей переменной. В сложившейся терминологии такие подьнтегральные функции называют выпуклыми интегрантами [1]. Недавно было доказано [2], что для интегральных функционалов в пространстве со сходимостью вьпуклость их интегрантов является и необходимым условием полунепрерьвности снизу.

При изучении вопросов существования минимума у интегральных функционалов с невыпукльми интегрантами в последние годы интенсивно развивается метод, основанный на применении теоремы А. А. Ляпунова о вьпуклости множества значений неатомической меры. В этом подходе функционал имеет вид

$$
\int_{\Omega} g(x, y(x)) d x+\int_{\Omega} h(x, u(x)) d x,
$$

где $g(x, y)$ вогнута по $y$, a $h(x, u)$ не является выпуклой по $u$. Основополагающей в этом направлении является работа [3], где рассмотрена классическая задача вариационного

Работа выполнена при частичной финансовой поддержке Российского фонда фундаментальных исследований, грант № 96-01-00326. 
исчисления с функционалом вида $(1), \Omega=[0, T]$, при связях, описьваемых линейными обыкновенными дифференциальными уравнениями в конечномерном пространстве, и граничных условиях. В дальнейшем идеи этой работы использовались для доказательства существования решений при соответствующих предположениях в задачах оптимального управления с функционалом вида (1) и с динамикой, описываемой линейными и нелинейными обькновенными дифференциальными уравнениями в конечномерном пространстве в [4]-[7]. Среди результатов этого типа следует отметить и ранние работы [8], [9]. Классические вариационные задачи с функционалом вида (1), зависящим от градиента и лапласиана, при тех или иных предположениях рассматривались в [10]-[13].

В работе [14] развивается другой подход, основанньй на использовании мер Юнга, при исследовании линейных управляемых систем с функционалами вида (1), где $g$ и $h$ квазивогнуты.

В настоящей работе доказьвается абстрактная теорема существования минимума функционала вида (1) на решениях систем, описываемых линейньми эллиптическими операторами. На примерах дается конкретизация этой теоремы для задач вариационного исчисления и оптимального управления.

1. Постановка задачи. Пусть $\Sigma$ - открытое или замкнутое ограниченное множество с конечной, неотрицательной, неатомической мерой Радона $\mu$. В приложениях в дальнейшем под $(\Sigma, \mu)$ мы будем понимать либо ограниченную открытую область $\Omega \mathrm{c}$ $n$-мерной мерой Лебега, либо ее гранищу $\partial \Omega$ с $(n-1)$-мерной мерой Лебега.

Пусть $V$ - гильбертово пространство и $a(y, \varphi)$ - непрерывная билинейная форма на $V \times V$. Тогда существует элемент $A y \in V^{\prime}$ такой, что $a(y, \varphi)=\langle A y, \varphi\rangle$ для всех $y, \varphi \in V$, где $A \in \mathscr{L}\left(V, V^{\prime}\right)$. Через $\mathscr{L}$ мы обозначаем соответствующие пространства непрерывных линейных операторов.

Пусть $W_{1}, W_{2}$ - банаховы пространства и $L_{1} \in \mathscr{L}\left(V, W_{1}\right), L_{2} \in \mathscr{L}\left(V, W_{2}\right)$. Пусть на $W_{1}$ и $U=L_{2}(\Sigma)^{k}$ заданы (не обязательно выпуклые) функционалы $G: W_{1} \rightarrow \mathbb{R} \cup\{+\infty\}$ и $H: U \rightarrow \mathbb{R} \cup\{+\infty\}$.

Рассмотрим измеримое многозначное отображение $\Gamma: \Sigma \rightarrow \mathbb{R}^{k}$ с непустыми замкнутыми (не обязательно выпуклыми) значениями. Обозначим через $S_{\Gamma}$ совокупность всех измеримых селекторов многозначного отображения $\Gamma$.

Рассмотрим задачу

$$
\min _{\substack{u \in U \cap S_{\Gamma} \\ y \in V}} G\left(L_{1} y\right)+H(u)
$$

при условиях

$$
\begin{gathered}
A y=\psi+B u, \quad y \in V, \quad \psi \in V^{\prime}, \\
F\left(L_{2} y\right) \leqslant \delta, \quad \delta \in \mathbb{R}^{l}, \quad \Phi(u) \leqslant \beta, \quad \beta \in \mathbb{R}^{m},
\end{gathered}
$$

где $B \in \mathscr{L}\left(U, V^{\prime}\right), \psi$ - фиксированный элемент из $V^{\prime}, F: W_{2} \rightarrow \mathbb{R}^{l}, \Phi: U \rightarrow \mathbb{R}^{m}$, а неравенства (4) понимаются покоординатно.

Всюду в дальнейшем предполагаем, что непрерьвная билинейная форма $a(y, \varphi)$ коэрцитивна, т.е.

$$
a(y, y) \geqslant \alpha\|y\|_{V}^{2}, \quad \alpha>0, \quad y \in V
$$


операторы $L_{1}$ и $L_{2}$ компактны, $F: W_{2} \rightarrow \mathbb{R}^{l}$ непрерьвна и ее компоненты являются вогнутыми функциями, $\Phi: U \rightarrow \mathbb{R}^{m}$ слабо полунепрерьвна снизу и ее компоненты являются вогнутыми функциями. Отображения $F$ и $\Phi$ будем назьвать вогнутыми.

Кроме того, пусть выполнены следующие предположения:

А) имеет место представление

$$
H(u)=\int_{\Sigma} h(x, u(x)) d \mu
$$

где $h: \Sigma \times \mathbb{R}^{k} \rightarrow \mathbb{R}$ - функция типа Каратеодори, почти всюду удовлетворяющая неравенству

$$
h(x, u) \geqslant \eta_{1}|u|^{2}-\eta_{2}(x), \quad \eta_{1}>0, \quad \eta_{2}(x) \geqslant 0, \quad \eta_{2} \in L_{1}(\Sigma)
$$

Б) $G: W_{1} \rightarrow \mathbb{R} \cup\{+\infty\}$ - вогнутая функция, удовлетворяющая неравенству

$$
G(w) \geqslant-\gamma_{1}|w|_{W_{1}}^{2}-\gamma_{2}, \quad \gamma_{1}>0, \quad \gamma_{2} \geqslant 0 .
$$

Из А) следует, что для любого $u \in U$ функция $h(x, u(x))$ измерима и ее неположительная часть интегрируема. Поэтому

$$
\int_{\Sigma} h(x, u(x)) d \mu
$$

либо конечен, либо равен $+\infty$.

ЗАмЕЧАнИЕ 1. В теории управления пространство $U$ назьвают пространством управлений, $V$ - пространством состояний, а $W_{1}$ и $W_{2}$ - пространствами наблюдений [15].

2. Релаксационная задача. Приведем ряд вспомогательных утверждений. При этом мы будем использовать результаты [1], которые были доказаны для случая, ког да $\Sigma$ - открытая ограниченная область в $\mathbb{R}^{n}$ с мерой Лебега. Отметим, что все результаты остаются справедливыми в нашем случае.

Рассмотрим функцию

$$
h_{\Gamma}(x, u)= \begin{cases}h(x, u), & \text { если } u \in \Gamma(x), \\ +\infty, & \text { если } u \notin \Gamma(x) .\end{cases}
$$

Обозначим через $h_{\Gamma}^{* *}(x, u)$ вторую сопряженную к $h_{\Gamma}(x, u)$ функцию [1].

ЛЕмма 1 [1]. Справедливы следующие утверәддения:

1) $h_{\Gamma}^{* *}(x, \cdot)$ - наибольшая выпуклая функиия, не превышающая $h_{\Gamma}(x, \cdot)$, m.е. почти всюду для любых и

$$
h_{\Gamma}^{* *}(x, u) \leqslant h_{\Gamma}(x, u)
$$

2) при выполнении условий роста (6)

$$
h_{\Gamma}^{* *}(x, u)=\min \left\{\sum_{i=1}^{k+1} \lambda_{i} h_{\Gamma}\left(x, \xi_{i}\right), u=\sum_{i=1}^{k+1} \lambda_{i} \xi_{i}: \lambda_{i} \geqslant 0, \sum_{i=1}^{k+1} \lambda_{i}=1\right\}
$$


3) для измеримой функиии и(· ) существуют измеримые функиии $p_{i}: \Omega \rightarrow[0,1]$, $v_{i}: \Omega \rightarrow \mathbb{R}^{k}, i=1, \ldots, k+1$, mакие, что

$$
\sum_{i=1}^{k+1} p_{i}(x)=1, \quad u(x)=\sum_{i=1}^{k+1} p_{i}(x) v_{i}(x), \quad h_{\Gamma}^{* *}(x, u(x))=\sum_{i=1}^{k+1} p_{i}(x) f\left(x, v_{i}(x)\right)
$$

Из свойств функции $h(x, u)$ и отображения $\Gamma$ следует, что для любого $u \in U$ функция $h_{\Gamma}(x, u)$ измерима. Следовательно, $h_{\Gamma}^{* *}(x, u)$ является нормальным интегрантом [1] и почти всюду для любых $u$

$$
h_{\Gamma}^{* *}(x, u) \geqslant \eta_{1}\|u\|^{2}-\eta_{2}(x)
$$

ЛЕмма 2 (лемма Лакса-Мильграма). Пусть билинейная непрерывная форма $a(y, \varphi)$ коэриитивна. Тогда для заданного $f \in V^{\prime}$ существует единственный әлемент $y \in V$, для которого $a(y, \varphi)=\langle f, \varphi\rangle, \varphi \in V$.

Отсюда следует, что для заданного $f \in V^{\prime}$ существует единственньй элемент $y \in V$ такой, что $A y=f$.

Рассмотрим вспомогательную задачу, обычно называемую релаксационной [1]:

$$
\min _{\substack{u \in U \\ y \in V}} G\left(L_{1} y\right)+H_{\Gamma}^{* *}(u)
$$

при ограничениях (3), (4), где

$$
H_{\Gamma}^{* *}(u)=\int_{\Sigma} h_{\Gamma}^{* *}(x, u(x)) d x
$$

Элемент $(u, y), u \in U, y \in V$, будем назьвать допустимым, если он удовлетворяет (3), (4). Из неравенств (7), (8) следует, что

$$
-\infty<G\left(L_{1} y\right)+H_{\Gamma}^{* *}(u) \leqslant+\infty .
$$

Поэтому для того чтобы задача (9) имела нетривиальное решение, мы должны предположить, что

В) существует допустимьй элемент $\left(u_{\text {доп }}, y_{\text {доп }}\right)$ такой, что

$$
G\left(L_{1} y_{\text {доп }}\right)+H_{\Gamma}^{* *}\left(u_{\text {доп }}\right)<+\infty .
$$

В противном случае любой допустимый элемент является решением задачи (9).

ТЕОРемА 1. Пусть выполнены предположения А)-В) $и$

$$
\eta_{1}-\frac{2 \gamma_{1}\left\|L_{1}\right\|^{2}\|B\|^{2}}{\alpha^{2}}>0
$$

Тогда релаксачионная задача (9) имеет решение. 
ДокАЗАТЕЛЬСТво. Из условия В) следует, что

$$
\inf _{\substack{u \in U \\ y \in V}} G\left(L_{1} y\right)+H_{\Gamma}^{* *}(u)<+\infty .
$$

Поэтому найдется число $M>0$ и последовательность $\left(u_{n}, y_{n}\right), n \geqslant 1$, допустимых элементов такие, что

$$
\begin{gathered}
\inf _{\substack{u \in U \\
y \in V}} G\left(L_{1} y\right)+H_{\Gamma}^{* *}(u)=\lim _{n \rightarrow \infty}\left(G\left(L_{1} y_{n}\right)+H_{\Gamma}^{* *}\left(u_{n}\right)\right)<+\infty, \\
G\left(L_{1} y_{n}\right)+H_{\Gamma}^{* *}\left(u_{n}\right) \leqslant M, \quad n \geqslant 1 .
\end{gathered}
$$

Из (7), (8) получим

$$
-\gamma_{1}\left\|L_{1}\right\|^{2}\left\|y_{n}\right\|^{2}-\gamma_{2}+\eta_{1}\left\|u_{n}\right\|^{2}-\int_{\Omega} \eta_{2}(x) d x \leqslant M .
$$

Пусть $z \in V$ таков, что

$$
a(z, \varphi)=\langle A z, \varphi\rangle=\langle f, \varphi\rangle, \quad \varphi \in V
$$

(существование такого $z$ обеспечивается леммой 2 ). Тогда, используя (3), (5), получим

$$
\alpha\left\|y_{n}\right\|^{2} \leqslant\|A\|\|z\|\left\|y_{n}\right\|+\|B\|\left\|u_{n}\right\|\left\|y_{n}\right\|
$$

или $\alpha\left\|y_{n}\right\| \leqslant\|A\|\|z\|+\|B\|\left\|u_{n}\right\|$. Следовательно,

$$
\left\|y_{n}\right\|^{2} \leqslant \frac{2}{\alpha^{2}}\left(\|A\|^{2}\|z\|^{2}+\|B\|^{2}\left\|u_{n}\right\|^{2}\right) .
$$

Из (10), (11), (12) получаем

$$
\left\|u_{n}\right\|^{2} \leqslant \frac{\alpha^{2}\left(M+\gamma_{2}+\left\|\eta_{2}\right\|_{L_{1}(\Omega)}\right)+2 \gamma_{1}\|A\|^{2}\left\|L_{1}\right\|^{2}\|z\|^{2}}{\alpha^{2} \eta_{1}-2 \gamma_{1}\left\|L_{1}\right\|^{2}\|B\|^{2}} .
$$

Из (13) следует, что последовательность $u_{n} \in U, n \geqslant 1$, ограничена. Аналогично, согласно (12) последовательность $y_{n} \in V, n \geqslant 1$, ограничена в $V$. Так как $U$ и $V$ рефлексивны, то можно выбрать подпоследовательности $u_{n_{j}}, y_{n_{j}}, j \geqslant 1$, последовательностей $u_{n}, y_{n}, n \geqslant 1$, слабо сходяшиеся к некоторым $u_{0}, y_{0}$ соответственно. Не ограничивая обшности, будем считать, что сами последовательности $u_{n}, y_{n}, n \geqslant 1$, слабо сходятся к $u_{0}, y_{0}$. Тогда из свойств операторов $A, B, L_{2}$ и отображений $F, \Phi$ следует, что соотношения (3), (4) выполняются для $u_{0}, y_{0}$.

Покажем, что функционал $G\left(L_{1} y\right)$ является непрерывным и вогнутым отображением из $U$ в $\mathbb{R}$. Вогнутость $G\left(L_{1} y\right)$ очевидна. Из условий А)-В) следует, что на некотором допустимом элементе $\left(u_{\text {доп }}, y_{\text {доп }}\right)$

$$
-\infty<-G\left(L_{1} y_{\text {доп }}\right) \quad \text { и } \quad-G\left(L_{1} y\right) \leqslant \gamma_{1}\left\|L_{1}\right\|^{2}\|y\|_{V}^{2}+\gamma_{2}
$$

для любых $y \in V$. Тогда из [1, предложение 2.5, c. 21]) следует, что $-G\left(L_{1} y\right)$ является непрерьвной функцией из $V$ в $\mathbb{R}$. Следовательно, таковой является и функция $G\left(L_{1} y\right)$. 
Так как оператор $A$ имеет непрерьвньй обратньй $A^{-1}: V^{\prime} \rightarrow V$, вьполнено $y_{n}=$ $A^{-1}\left(f+B u_{n}\right)$. Поэтому из слабой сходимости $u_{n}$ к $u_{0}$ следует слабая сходимость $y_{n}$ к $y_{0}$. Так как оператор $L_{1}$ компактен, переходя к подпоследовательности $\left(u_{n_{j}}, y_{n_{j}}\right)$, получим

$$
G\left(L_{1} y_{0}\right)+\underline{\lim }_{j \rightarrow \infty} H_{\Gamma}^{* *}\left(u_{n_{j}}\right)=\lim _{n \rightarrow \infty}\left(G\left(L_{1} y_{n}\right)+H_{\Gamma}^{* *}\left(u_{n}\right)\right) .
$$

Воспользовавшись $(8)$, свойствами функции $h_{\Gamma}^{* *}(x, u)$ и теоремой 2.1 из [1, с. 242], получаем

$$
H_{\Gamma}^{* *}\left(u_{0}\right) \leqslant \varliminf_{j \rightarrow \infty} H_{\Gamma}^{* *}\left(u_{n_{j}}\right)
$$

Из (14), (15) следует, что

$$
G\left(L_{1} y_{0}\right)+H_{\Gamma}^{* *}\left(u_{0}\right)=\inf _{\substack{u \in U \\ y \in V}} G\left(L_{1} y\right)+H_{\Gamma}^{* *}(u) .
$$

Теорема доказана.

3. Основной результат. Как и в релаксационной задаче, для того чтобы задача (2) имела нетривиальное решение, мы должны предположить, что

$\Gamma)$ существует допустимьй элемент $\left(u_{\text {доп }}, y_{\text {доп }}\right)$ такой, что

$$
G\left(L_{1} y_{\text {доп }}\right)+H\left(u_{\text {доп }}\right)<+\infty \text {. }
$$

ТЕОрема 2. Пусть выполнены предположсения А), Б), Г) и неравенство (10). Тогда задача (2) имеет решение.

ДокАЗАТЕЛЬСтво. Из А), Б), Г) следует, что для некоторого допустимого элемента $\left(u_{\text {доп }}, y_{\text {доп }}\right)$ задачи $(2)$ будет иметь место неравенство

$$
H\left(u_{\text {доп }}\right)<+\infty, \quad u_{\text {доп }} \in S_{\Gamma} .
$$

Поэтому $h_{\Gamma}\left(x, u_{\text {доп }}(x)\right)=h\left(x, u_{\text {доп }}(x)\right)$ почти всюду. Тогда в силу $(8)$

$$
G\left(L_{1} y_{\text {доп }}\right)+H_{\Gamma}^{* *}\left(u_{\text {доп }}\right) \leqslant G\left(L_{1} y_{\text {доп }}\right)+H\left(u_{\text {доп }}\right)<+\infty .
$$

Следовательно, для задачи (9) будет сушествовать оптимальное решение $\left(u_{0}, y_{0}\right)$.

Пусть

$$
H_{\Gamma}(u)=\int_{\Sigma} h_{\Gamma}(x, u(x)) d \mu
$$

Рассмотрим задачу

$$
\min _{\substack{u \in U \cap S_{\Gamma} \\ y \in V}} G\left(L_{1} y\right)+H_{\Gamma}(u)
$$

при ограничениях $(3),(4)$, которая эквивалентна исходной задаче (2). Поскольку

$$
G\left(L_{1} y_{0}\right)+H_{\Gamma}^{* *}\left(u_{0}\right) \leqslant \inf _{\substack{u \in U \cap S_{\Gamma} \\ y \in V}} G\left(L_{1} y\right)+H_{\Gamma}(u)
$$


теорема будет доказана, если мы покажем, что существует допустимый элемент $\left(u_{*}, y_{*}\right)$ такой, что

$$
G\left(L_{1} y_{0}\right)+H_{\Gamma}^{* *}\left(u_{0}\right)=G\left(L_{1} y_{*}\right)+H_{\Gamma}\left(u_{*}\right)
$$

Так как

$$
-\infty<\int_{\Sigma} h_{\Gamma}^{* *}\left(x, u_{0}(x)\right) d \mu<+\infty
$$

функция $h_{\Gamma}^{* *}\left(x, u_{0}(x)\right)$ интегрируема и почти всюду

$$
\left|h_{\Gamma}^{* *}\left(x, u_{0}(x)\right)\right|<\infty .
$$

Воспользовавшись утверждением 3) леммы 1 , получим, что существуют измеримые функции $p_{i}: \Sigma \rightarrow[0,1], v_{i}: \Sigma \rightarrow \mathbb{R}^{k}, i=1, \ldots, k+1$, такие, что

$$
\begin{aligned}
& \sum_{i=1}^{k+1} p_{i}(x)=1, \quad u_{0}(x)=\sum_{i=1}^{k+1} p_{i}(x) v_{i}(x), \\
& h_{\Gamma}^{* *}\left(x, u_{0}(x)\right)=\sum_{i=1}^{k+1} p_{i}(x) h_{\Gamma}\left(x, v_{i}(x)\right) .
\end{aligned}
$$

Из $(16),(18)$ получаем, что функция $h_{\Gamma}\left(x, v_{i}(x)\right)$ может принимать на множестве $E$ положительной меры значение $+\infty$ только в том случае, если $p_{i}(x)=0$ на этом множестве. Тогда, не меняя $(17),(18)$, можно полагать, что $v_{i}(x) \in \Gamma(x)$ на множестве $E$. Если же $p_{i}(x)>0$ на $E$, то $v_{i}(x) \in \Gamma(x), i=1, \ldots, k+1$. Тогда согласно (18)

$$
h_{\Gamma}^{* *}\left(x, u_{0}(x)\right)=\sum_{i=1}^{k+1} p_{i}(x) h\left(x, v_{i}(x)\right)
$$

и $u_{0}(x) \in \overline{\mathrm{co}} \Gamma(x)$ при почти всех $x \in \Sigma$.

Согласно теореме Лузина [1] существует последовательность $K_{j}, j \geqslant 1$, компактных, попарно не пересекающихся множеств таких, что $\mu\left(\bigcup_{j=1}^{\infty} K_{j}\right)=\mu(\Sigma)$, и сужения $v_{i}(x)$, $h\left(x, v_{i}(x)\right), i=1, \ldots, k+1$, на $K_{j}$ непрерывны. Рассмотрим измеримое многозначное отображение $\Gamma_{1}: \Sigma \rightarrow \mathbb{R}$ с компактными значениями, определенное по правилу

$$
\Gamma_{1}(x)=\bigcup_{i=1}^{k+1} h\left(x, v_{i}(x)\right)
$$

Тогда почти всюду $h_{\Gamma}^{* *}\left(x, u_{0}(x)\right) \in \operatorname{co} \Gamma_{1}(x)$. Так как на каждом из множеств $K_{j}$ многозначное отображение $\Gamma_{1}$ в силу непрерьвности $h\left(x, v_{i}(x)\right)$ на $K_{j}$ интегрально ограничено, в соответствии с теоремой 1 из [16, с. 349]

$$
\int_{K_{j}} \Gamma_{1}(x) d \mu=\int_{K_{j}} \operatorname{co} \Gamma_{1}(x) d \mu
$$

Отсюда следует, что для каждого $j \geqslant 1$ существует разбиение $K_{j}$ на попарно непересекающиеся измеримые множества $K_{j}^{i}, i=1, \ldots, k+1$, со свойством

$$
\int_{K_{j}} h_{\Gamma}^{* *}\left(x, u_{0}(x)\right) d x=\int_{K_{j}} \sum_{i=1}^{k+1} p_{i}(x) h\left(x, v_{i}(x)\right) d x=\int_{K_{j}} \sum_{i=1}^{k+1} \chi_{K_{j}^{i}}(x) h\left(x, v_{i}(x)\right) d x
$$


где $\chi_{K_{j}^{i}}(x)$ - характеристическая функция множества $K_{j}^{i}$.

Покажем, что для любого разбиения $K_{j}^{i}$ множества $K_{j}$, для которого справедливо равенство (20), функция

$$
w(x)=\sum_{j=1}^{\infty} \sum_{i=1}^{k+1} \chi_{K_{j}^{i}}(x) h\left(x, v_{i}(x)\right)
$$

интегрируема. Как следствие из (6) получим, что функция

$$
v(x)=\sum_{j=1}^{\infty} \sum_{i=1}^{k+1} \chi_{K_{j}^{i}}(x) v_{i}(x)
$$

интегрируема с квадратом.

Рассмотрим последовательность

$$
s_{n}(x)=\sum_{j \leqslant n} \sum_{i=1}^{k+1} \chi_{K_{j}^{i}}(x)\left(h\left(x, v_{i}(x)\right)+\eta_{2}(x)\right) .
$$

Согласно (6) последовательность $s_{n}(x)$ неотрицательна, монотонно не убьвает и почти всюду сходится к $w(x)+\eta_{2}(x)$. Воспользовавшись $(20),(21)$, получаем

$$
\begin{aligned}
\int_{\Sigma} s_{n}(x) d x & =\sum_{j \leqslant n} \int_{K_{j}} \sum_{i=1}^{k+1} \chi_{K_{j}^{i}}(x)\left(h\left(x, v_{i}(x)\right)+\eta_{2}(x)\right) d x \\
& =\sum_{j \leqslant n} \int_{K_{j}} \sum_{i=1}^{k+1} p_{i}(x)\left(h\left(x, v_{i}(x)\right)+\eta_{2}(x)\right) d x \\
& \leqslant \int_{\Sigma}\left(h_{\Gamma}^{* *}\left(x, u_{0}(x)\right)+\eta_{2}(x)\right) d x<+\infty .
\end{aligned}
$$

Теперь из (22) и теоремы Фату [17, с. 542] вытекает, что

$$
\lim _{n \rightarrow \infty} \int_{\Sigma} s_{n}(x) d x=\int_{\Sigma} \lim _{n \rightarrow \infty} s_{n}(x) d x=\int_{\Sigma}\left(w(x)+\eta_{2}(x)\right) d x<\infty .
$$

Следовательно, функция $w(x)$ интегрируема.

При доказательстве теоремы 2 было установлено, что функционал $G\left(L_{1} y\right)$ является непрерьвньм вогнутым отображением из $V$ в $\mathbb{R}$. Тогда функционал $G_{1}(u)=G\left(L_{1} A^{-1} \times\right.$ $(f+B u))$, где $A^{-1}$ - обратньй к $A$ оператор, является непрерывным вогнутым отображением из $U$ в $\mathbb{R}$. Аналогично, $F_{1}: U \rightarrow \mathbb{R}^{l}, F_{1}(u)=F\left(L_{2} A^{-1}(f+B u)\right)$ является непрерьвньп вогнутым отображением из $U$ в $\mathbb{R}^{l}$.

Рассмотрим непрерьвные вьпуклые отображения $-G_{1}(u),-F_{1}(u)$ и полунепрерьвное снизу в слабой топологии пространства $U$ вьпуклое отображение $-\Phi(u)$ из $U$ в $\mathbb{R}^{m}$. В соответствии с предложением 2.5 из $[1$, с. 21] отображение $-\Phi(u)$ будет непрерывным выпуклым отображением в слабой топологии пространства $V$. Согласно предложению 5.2 из $[1$, с. 31$]$ отображения $-G_{1}(u),-F_{1}(u)$ и $-\Phi(u)$ субдифференцируемы в 
точке $u_{0}$, т.е. существуют элементы $g^{\prime} \in U^{\prime}=L_{2}(\Sigma), f^{\prime} \in L_{2}(\Sigma)^{l}, \phi^{\prime} \in L_{2}(\Sigma)^{m}$ такие, что

$$
\begin{gathered}
\int_{\Sigma}\left\langle g^{\prime}(x), u(x)-u_{0}(x)\right\rangle d \mu \leqslant-G_{1}(u)+G_{1}\left(u_{0}\right), \\
\int_{\Sigma}\left\langle f^{\prime}(x), u(x)-u_{0}(x)\right\rangle d \mu \leqslant-F_{1}(u)+F_{1}\left(u_{0}\right), \\
\int_{\Sigma}\left\langle\phi^{\prime}(x), u(x)-u_{0}(x)\right\rangle d \mu \leqslant-\Phi(u)+\Phi\left(u_{0}\right)
\end{gathered}
$$

для всех $u \in U . \mathrm{B}(24)$ скалярные произведения и соответственно неравенства понимаются покомпонентно.

Введем пространство $X=\mathbb{R}^{l+m+2}$. Тогда на $\Sigma$ определены измеримые функции $\gamma_{i}: \Sigma \rightarrow X, i=1, \ldots, k+1$, с компонентами

$$
\gamma_{i}(x)=\left\{\left\langle f^{\prime}(x), v_{i}(x)\right\rangle,\left\langle\phi^{\prime}(x), v_{i}(x)\right\rangle,\left\langle g^{\prime}(x), v_{i}(x)\right\rangle, h\left(x, v_{i}(x)\right)\right\}
$$

Рассмотрим измеримое многозначное отображение $\Gamma^{*} \rightarrow X, \Gamma^{*}(x)=\left\{\bigcup_{i=1}^{k+1} \gamma_{i}(x)\right\}$, $x \in \Sigma$, с замкнутыми значениями. Тогда из $(17),(19)$ следует, что функция

$$
\gamma(x)=\left\{\left\langle f^{\prime}(x), u_{0}(x)\right\rangle,\left\langle\phi^{\prime}(x), u_{0}(x)\right\rangle,\left\langle g^{\prime}(x), u_{0}(x)\right\rangle, h_{\Gamma}^{* *}\left(x, u_{0}(x)\right)\right\}
$$

почти всюду удовлетворяет включению $\gamma(x) \in \operatorname{co} \Gamma^{*}(x)$. На каждом из множеств $K_{j}$ отображение со $\Gamma^{*}(x)$ интегрально ограничено. Тогда, используя свойства многозначного интеграла (см. $[16$, теорема 1, c. 349$])$, получаем, что для каждого $K_{j}$ существует разбиение $K_{j}^{i}, i=1, \ldots, k+1$, такое, что

$$
\begin{aligned}
\int_{K_{j}}\left\langle f^{\prime}(x), u_{0}(x)\right\rangle d \mu & =\int_{K_{j}} \sum_{i=1}^{k+1} \chi_{K_{j}^{i}}(x)\left\langle f^{\prime}(x), v_{i}(x)\right\rangle d \mu, \\
\int_{K_{j}}\left\langle\phi^{\prime}(x), u_{0}(x)\right\rangle d \mu & =\int_{K_{j}} \sum_{i=1}^{k+1} \chi_{K_{j}^{i}}(x)\left\langle\phi^{\prime}(x), v_{i}(x)\right\rangle d \mu \\
\int_{K_{j}}\left\langle g^{\prime}(x), u_{0}(x)\right\rangle d \mu & =\int_{K_{j}} \sum_{i=1}^{k+1} \chi_{K_{j}^{i}}(x)\left\langle g^{\prime}(x), v_{i}(x)\right\rangle d \mu \\
\int_{K_{j}} h_{\Gamma}^{* *}\left(x, u_{0}(x)\right) d \mu & =\int_{K_{j}} \sum_{i=1}^{k+1} \chi_{K_{j}^{i}}(x) h\left(x, v_{i}(x)\right) d \mu .
\end{aligned}
$$

Определим измеримые функции

$$
u_{*}(x)=\sum_{j=1}^{\infty} \sum_{i=1}^{k+1} \chi_{K_{j}^{i}}(x) v_{i}(x), \quad w(x)=\sum_{j=1}^{\infty} \sum_{i=1}^{k+1} \chi_{K_{j}^{i}}(x) h\left(x, v_{i}(x)\right)
$$


Тогда $u_{*}(x) \in \Gamma(x)$ почти всюду. Из равенства (26), как было доказано выше, следует, что функции $\left|u_{*}(x)\right|^{2}$ и $w(x)$ интегрируемы. Тогда с учетом интегрируемости функций $\left|u_{*}(x)\right|^{2}$ и $h_{\Gamma}^{* *}\left(x, u_{0}(x)\right)$ из $(25),(26)$ получаем

$$
\begin{gathered}
\int_{\Sigma}\left\langle f^{\prime}(x), u_{0}(x)\right\rangle d \mu=\int_{\Sigma}\left\langle f^{\prime}(x), u_{*}(x)\right\rangle d \mu, \quad \int_{\Sigma}\left\langle\phi^{\prime}(x), u_{0}(x)\right\rangle d \mu=\int_{\Sigma}\left\langle\phi^{\prime}(x), u_{*}(x)\right\rangle d \mu \\
\int_{\Sigma}\left\langle g^{\prime}(x), u_{0}(x)\right\rangle d \mu=\int_{\Sigma}\left\langle g^{\prime}(x), u_{*}(x)\right\rangle d \mu \\
H_{\Gamma}^{* *}\left(u_{0}\right)=\int_{\Sigma} h\left(x, u_{*}(x)\right) d \mu=H\left(u_{*}\right)
\end{gathered}
$$

Обозначим через $y_{*} \in V$ элемент $y_{*}=A^{-1}\left(f+B u_{*}\right)$. Тогда

$$
A y_{*}=f+B u_{*} .
$$

Воспользовавшись $(23),(24)$ и (27), получаем

$$
\begin{gathered}
G\left(L_{1} y_{*}\right) \leqslant G\left(L_{1} y_{0}\right), \\
F\left(L_{2} y_{*}\right) \leqslant F\left(L_{2} y_{0}\right) \leqslant \delta, \quad \Phi\left(u_{*}\right) \leqslant \Phi\left(u_{0}\right) \leqslant \beta .
\end{gathered}
$$

Из (28), (30) следует, что

$$
G\left(L_{1} y_{*}\right)+H\left(u_{*}\right)=G\left(L_{1} y_{*}\right)+H_{\Gamma}^{* *}\left(u_{0}\right) \leqslant G\left(L_{1} y_{0}\right)+H_{\Gamma}^{* *}\left(u_{0}\right) .
$$

Поскольку $u_{*}(x) \in \Gamma(x)$, то

$$
h_{\Gamma}^{* *}\left(x, u_{*}(x)\right) \leqslant h_{\Gamma}\left(x, u_{*}(x)\right)=h\left(x, u_{*}(x)\right) .
$$

Поэтому, воспользовавшись (32), получим

$$
G\left(L_{1} y_{*}\right)+H_{\Gamma}^{* *}\left(u_{*}\right) \leqslant G\left(L_{1} y_{*}\right)+H\left(u_{*}\right) \leqslant G\left(L_{1} y_{0}\right)+H_{\Gamma}^{* *}\left(u_{0}\right) .
$$

Так как $\left(u_{0}, y_{0}\right)$ - решение задачи $(9)$, из $(29),(31),(33)$ следует, что

$$
G\left(L_{1} y_{*}\right)+H\left(u_{*}\right)=G\left(L_{1} y_{0}\right)+H_{\Gamma}^{* *}\left(u_{0}\right)
$$

Тем самьм, $\left(u_{*}, y_{*}\right)$ является решением задачи (2). Теорема доказана.

ЗАмЕчАниЕ 2. При доказательстве интегрируемости функции $w(x)$ использовались идеи работы [3].

ЗАмЕчАниЕ 3. При доказательстве теоремы мы не использовали в явном виде теорему Ляпунова о выпуклости значений векторной меры. Однако, она заложена в свойствах многозначного интеграла, которые мы использовали. 
4. Примеры. Пусть $\Omega$ - открытая ограниченная область из $\mathbb{R}^{n}$ с мерой Лебега $d x$ и кусочно-липшицевой границей, $g: \Omega \times \mathbb{R}^{N} \rightarrow \mathbb{R}$ - функция типа Каратеодори и

Д) $g(x, z)$ при почти всех $x$ вогнута по $z$ и

$$
g(x, z) \geqslant-\gamma_{1}|z|^{2}-\gamma_{2}(x), \quad \gamma_{1}>0, \quad \gamma_{2} \in L_{2}(\Omega), \quad \gamma_{2}(x) \geqslant 0 .
$$

Из $[1$, следствие 2.3, с. 22$]$ следует, что $g(x, z)$ при почти всех $x$ непрерывна по $z$. Определим на $L_{2}(\Omega)^{N}=W_{1}$ функционал

$$
G(z)=\int_{\Omega} g(x, z(x)) d x .
$$

Из (34) следует, что $G: W_{1} \rightarrow \mathbb{R} \cup\{+\infty\}$ удовлетворяет условию Б).

Рассмотрим на некотором пространстве $V \times V$ непрерьвную билинейную форму

$$
a(y, \varphi)=\sum_{i, j=1}^{n} \int_{\Omega} a_{i j}(x) \frac{\partial y}{\partial x_{i}} \frac{\partial \varphi}{\partial x_{j}} d x+\int_{\Omega} a_{0} y \varphi d x
$$

где

$$
\begin{gathered}
\sum_{i, j=1}^{n} a_{i j}(x) \xi_{i} \xi_{j} \geqslant \alpha|\xi|^{2} \quad \text { для всех } \xi \in \mathbb{R}^{n} \text { почти всюду в } \Omega, \\
a_{i j}, a_{0} \in L^{\infty}, \quad a_{0}(x) \geqslant \alpha>0 \text { почти всюду в } \Omega .
\end{gathered}
$$

Определим формальный линейньй эллиптический оператор $A$ второго порядка:

$$
A y=-\sum_{i, j=1}^{n} \frac{\partial}{\partial x_{i}}\left(a_{i j}(x) \frac{\partial y}{\partial x_{j}}\right)+a_{0} y .
$$

ПримеР 1 (граничное управление системой, описьваемой задачей Неймана). Пусть $V=H^{1}(\Omega), V^{\prime}=H^{-1}(\Omega), U=L_{2}(\Sigma)$, где $\Sigma$ - граница множества $\Omega$ с $(n-1)$-й мерой Лебега $d \sigma$.

Пусть $g: \Omega \times \mathbb{R} \rightarrow \mathbb{R}, h: \Sigma \times \mathbb{R} \rightarrow \mathbb{R}, \Gamma: \Sigma \rightarrow \mathbb{R}$ - измеримое многозначное отображение с замкнутыми значениями, $F: L_{2}(\Omega) \rightarrow \mathbb{R}^{l}$ и $\Phi: L_{2}(\Sigma) \rightarrow \mathbb{R}^{m}$ - отображения со свойствами, указанными в п. 1 , оператор $B \in \mathscr{L}\left(L_{2}(\Sigma), H^{-1}(\Omega)\right)$ определен по формуле

$$
\langle B u, \varphi\rangle=\int_{\Sigma} u \varphi d \sigma
$$

$L_{1}=L_{2}-$ операторы вложения $V$ в $L_{2}(\Omega)$.

Рассмотрим задачу

$$
\min _{\substack{u \in L_{2}(\Sigma) \cap S_{\Gamma} \\ y \in L_{2}(\Omega)}} \int_{\Omega} g\left(x, L_{1} y(x)\right) d x+\int_{\Sigma} h(s, u(s)) d \sigma
$$

при ограничениях

$$
\begin{gathered}
a(y, \varphi)=\langle\psi, \varphi\rangle+\langle B u, \varphi\rangle, \quad \varphi \in H^{1}(\Omega), \\
F\left(L_{2} y\right) \leqslant \delta, \quad \delta \in \mathbb{R}^{l}, \quad \Phi(u) \leqslant \beta, \quad \beta \in \mathbb{R}^{m} .
\end{gathered}
$$


Уравнение (38) представляет собой задачу Неймана

$$
A y=\psi_{1}, \quad x \in \Omega, \quad \frac{\partial y}{\partial \nu_{A}}=\psi_{2}+u, \quad x \in \Sigma,
$$

где $u \in U$ - управление, $y$ - состояние, $A$ - оператор вида $(36), \psi_{1}$ и $\psi_{2}$ - заданные элементы $L_{2}(\Omega)$ и $L_{2}(\Sigma)$ соответственно, $\partial y / \partial \nu_{A}-$ производная по направлению внешней нормали к границе области $\Omega$,

$$
\langle\psi, \varphi\rangle=\int_{\Omega} \psi_{1} \varphi d x+\int_{\Sigma} \psi_{2} \varphi d \sigma
$$

ТЕОРемА 3. Пусть выполнены условия А), Д), (10) и для задачи (37) существует допустимый әлемент. Тогда задача (37) с условиями (38), (39) имеет решение.

Результат вытекает из теоремы 2 и компактности вложения $V$ в $L_{2}(\Omega)$, поскольку $(38)$ определяет операторное уравнение $A y=\psi+B u$.

ПРимеР 2 (классическая задача вариационного исчисления без предположений выпуклости). Пусть $\Omega$ - ограниченная открытая область с границей класса $C^{2}, V=$ $H_{0}^{1}(\Omega) \cap H^{2}(\Omega)$. Рассмотрим задачу минимизации функционала

$$
\min _{\substack{y \in V \\ A y \in L_{2}(\Omega) \cap S_{\Gamma}}} \int_{\Omega} g\left(x, y(x), \frac{\partial y}{\partial x_{1}}(x), \ldots, \frac{\partial y}{\partial x_{n}}(x)\right) d x+\int_{\Omega} h(x, A y(x)) d x
$$

при ограничениях

$$
\begin{gathered}
F(y)=\int_{\Omega} f\left(x, y(x), \frac{\partial y}{\partial x_{1}}(x), \ldots, \frac{\partial y}{\partial x_{n}}(x)\right) d x \leqslant \delta, \quad \delta \in \mathbb{R}^{l}, \\
\Phi(y)=\int_{\Omega}\langle\phi(x), A y(x)\rangle d x \leqslant \beta, \quad \beta \in \mathbb{R}^{m} .
\end{gathered}
$$

Здесь производные понимаются в обобщенном смысле, $g: \Omega \times \mathbb{R}^{n+1} \rightarrow \mathbb{R}$ удовлетворяет условию Д), $h: \Omega \times \mathbb{R} \rightarrow \mathbb{R}$ удовлетворяет условию $\mathrm{A}), \Gamma: \Omega \rightarrow \mathbb{R}$ - измеримое многозначное отображение с замкнутыми значениями, $f: \Omega \times \mathbb{R}^{n+1} \rightarrow \mathbb{R}^{l}$-отображение типа Каратеодори, компоненты которого при почти всех $x$ вогнуты и удовлетворяют условиям роста

$$
f_{i}(x, z) \geqslant-\mu_{i}(x)|z|^{2}-\nu_{i}(x), \quad i=1, \ldots, l,
$$

где $\mu_{i}(x) \geqslant 0, \nu_{i}(x) \geqslant 0, \mu_{i} \in L_{\infty}(\Omega), \nu_{i} \in L_{1}(\Omega), \phi \in L_{2}(\Omega)^{m}, A$ - эллиптический оператор вида (36) с коэффициентами, удовлетворяюшими $(35)$, и, кроме того, $a_{i j}$ равномерно липшицевы в $\Omega$.

Обозначим через $L_{1}: V \rightarrow L_{2}(\Omega)^{n+1}$ компактньй оператор, определенньй по правилу

$$
L_{1}: y \mapsto L_{1} y=\left\{y, \frac{\partial y}{\partial x_{1}}, \ldots, \frac{\partial y}{\partial x_{n}}\right\}
$$


Теорема 4. Пусть выполнены условия А), Д), (41), (10), где $B=I$ - тохдественный оператор, и существует допустимый әлемент. Тогда классическая задача вариачионного исчисления (40) имеет решение.

ДокАЗАТЕЛЬСтво. Из неравенства (41) и существования допустимого элемента с помощью рассуждений, проведенных в теореме 1 для доказательства непрерывности отображения $G\left(L_{1} y\right)$, получаем, что $F_{i}(y), i=1, \ldots, l$, являются вогнутыми непрерывными отображениями из $L_{2}(\Omega)^{n+1}$ в $\mathbb{R}$, а условие $\phi \in L_{2}(\Omega)^{m}$ означает, что

$$
\int_{\Omega}\langle\phi(x), A y(x)\rangle d x
$$

- система непрерывных линейных функционалов на $L_{2}(\Omega)$. Теперь результат следует из теоремы 2 , если учесть, что в силу теоремы о регулярности решения [18] обобщенная задача Дирихле

$$
A y=u, \quad y \in H_{0}^{1}(\Omega), \quad u \in L_{2}(\Omega),
$$

имеет единственное решение в классе $V$.

\section{СПИСОК ЦИТИРОВАННОЙ ЛИТЕРАТУРЫ}

[1] Экланд И., Темам Р. Выпуклый анализ и вариационные проблемы. М.: Мир, 1979.

[2] Сычев М. А. Необходимые и достаточные условия в теоремах полунепрерывности и сходимости с функционалом // Матем. сб. 1995. Т. 186. №6. С. 77-108.

[3] Cellina A., Colombo G. On a classical problem of the calculus of variations without convexity assumptions // Ann. Inst. H. Poincaré. Anal. Non Linéaire. 1990. V. 7. № 2. P. 97-106.

[4] Raymond J.P. Existence theorems in optimal control problems without convexity assumptions // J. Optim. Theory Appl. 1990. V. 67. P. 109-132.

[5] Mariconda C. A generalization of the Cellina-Colombo theorem for a class of nonconvex variational problems // J. Math. Anal. Appl. 1993. V. 1975. P. 514-522.

[6] Colombo G., Goncharov V. V. Existence for a nonconvex optimal control problem with nonlinear dynamics // Nonlinear Anal. 1995. V. 24. №6. P. 795-800.

[7] Colombo G., Goncharov V. V. On a class of nonconvex and nonlinear optimal control problems // NoDEA Nonlinear Differential Equations Appl. 1996. V. 3. P. 115-126.

[8] Olech C. Integrals of set-valued functions and linear optimal control problems // Colloque sur la Théorie Mathématique du Controle Optimal. Louvain: CBRM, Vander, 1970. P. 109-125.

[9] Chesari L. Optimization Theory and Applications. New York: Springer, 1983.

[10] Cellina A. On minima of a functional of the gradient: sufficient conditions // Nonlinear Anal. 1993. V. 20. P. 343-347.

[11] Cellina A., Perrotta S. On minima of radially symmetric functionals of the gradient // Nonlinear Anal. 1994. V. 23. P. 239-249.

[12] Flores F. On Radial Solutions to Non-Convex Variational Problems. Preprint SISSA, 1991. Ref. 127/M.

[13] Cellina A. Minimizing a Functional Depending on $\nabla u$ and on $u$. Preprint SISSA, 1995. Ref. 41/95/M.

[14] Balder E. New existence results for optimal control in the absence of convexity: the importance of extremality // SIAM J. Control Optim. 1994. V. 32. №3. P. 890-916.

[15] Лионс ЖК.-Л. Оптимальное управление системами, описываемыми уравнениями с частными производными. М.: Мир, 1972.

[16] Иоффе А. Д., Тихомиров В. М. Теория экстремальных задач. М.: Наука, 1974.

[17] Шварц Л. Анализ. Т. 1. М.: Мир, 1972.

[18] Гилбарг Д., Трудингер Н. Эллиптические дифференциальные уравнения с частными производными второго порядка. М.: Наука, 1989. 